\title{
ENSINO DA LÍNGUA INGLESA PARA SURDOS DESDE UMA PERSPECTIVA DE PRÁTICAS TRANSLÍNGUES: MOTIVAÇÕES E INTERESSES
}

\author{
ENSEÑANZA DEL INGLÉS PARA SORDOS DESDE UNA PERSPECTIVA DE \\ PRÁCTICAS TRANSLINGÜES: MOTIVACIONES E INTERESES
}

\author{
THE TEACHING OF ENGLISH FOR THE DEAF TRHOUGH THE PERSPECTIVE OF \\ TRANSLINGUAL PRACTICES: MOTIVATIONS AND INTERESTS
}

\author{
Jorge Armando BECERRA-CALERO ${ }^{1}$ \\ Angélica María ROJAS-ISAZA ${ }^{2}$
}

RESUMO: O artigo a seguir teve como objetivo analisar os processos linguísticos e de assimilação que facilitaram a aprendizagem das estruturas básicas do inglês escrito em estudantes surdos. A pesquisa se enquadrou na metodologia denominada Investigación Acción Participativa (FALS BORDA, 1987), e foi desenvolvida com uma mostra correspondente a seis alunos surdos de ensino meio, falantes da língua de sinais, em uma instituição pública de Armênia, na Colômbia. Os dados foram coletados em 20 intervenções durante um semestre acadêmico, por intermédio de gravações de vídeo e entrevistas. As aulas foram feitas tomando como referência o nível morfossintático gramatical do inglês, correspondente ao nível A1 do Marco Comum Europeu, e focalizadas na perspectiva metodológica de práticas translíngues (CANAGARAJAH, 2013; GARCÍA; WEI, 2014). As conclusões deste trabalho mostraram que não só se relacionam aspectos cognitivos ao processo de aprendizagem, senão que este envolve necessidades, usos, relações e adaptações aos novos conhecimentos.

PALAVRAS-CHAVE: Estudantes surdos. Inglês língua estrangeira. Práticas translíngues. Língua de sinais. Nível morfossintático.

RESUMEN: El siguiente artículo tuvo como objetivo analizar los procesos lingüísticos y de asimilación que facilitaron el aprendizaje de las estructuras básicas del inglés escrito en estudiantes sordos. La investigación se enmarcó en la metodología Investigación Acción Participativa (FALS BORDA, 1987), desarrollado con una muestra correspondiente a seis estudiantes de grado 11, pertenecientes a la población sorda hablante de la lengua de señas, en una institución pública de Armenia, Colombia. Para recolectar los datos se llevaron a cabo 20 intervenciones durante un semestre académico, por medio de grabaciones y entrevistas. Estas se enfocaron en el nivel morfosintáctico gramatical del inglés, correspondiente a la escala Al en el Marco Común Europeo, enfocada en la perspectiva metodológica de prácticas translingües (CANAGARAJAH, 2013; GARCÍA; WEI, 2014). Las conclusiones de dicho trabajo arrojaron detalles que no solo relacionan lo cognitivo en este

1 Universidade Estadual de Mato Grosso (UNEMAT), Campo Grande - MS - Brasil. Doutorando em Linguística. ORCID: <https://orcid.org/0000-0001-7394-2654>. E-mail: jabecerrac@uqvirtual.edu.co

${ }^{2}$ Universidad de la Sabana (UNISABANA), Bogotá, D.C., Colombia. Profesora e investigadora de la Facultad de Educación. ORCID: <https://orcid.org/0000-0003-3059-9483>.E-mail: angeliza.rojas@ unisabana.edu.co 
proceso de aprendizaje, sino que van más allá, involucrando necesidades, usos, relaciones y adaptaciones a los nuevos saberes.

PALABRAS CLAVE: Estudiantes sordos. Inglés lengua extranjera. Prácticas translingües. Lengua de señas. Nivel morfosintáctico.

ABSTRACT: This article is the result of a research work framed within the methodology called Participatory Action Research (FALS BORDA, 1987). It selected a sample corresponding to six students of grade 11, belonging to the deaf population that uses sign language in a public institution in Armenia, Colombia. The research aimed to analyze the linguistic and assimilation processes that facilitated the learning of the basic structures of written English. To collect data, 20 interventions were carried out during one academic semester. These focused on the morphosyntactic grammatical level of English corresponding to the Al scale in the Common European Framework, through the translingual practices perspective (CANAGARAJAH, 2013; GARCÍA; WEI, 2014). The conclusions of this work showed details that not only relate to the cognitive process, but also go beyond, involving needs, uses, associations and adaptations of deaf individuals to new knowledge.

KEYWORDS: Deaf students. English foreign language. Translingual practices. Sign Language. Morphosyntactic level.

\section{Introducción}

En los últimos años se ha generado un creciente interés en la enseñanza y el aprendizaje de las lenguas extranjeras. El inglés, como lengua empleada por los motores del proceso de globalización, ocupa un lugar predominante. Dentro de estos procesos, es posible identificar necesidades y capacidades lingüísticas de los nuevos hablantes, así como técnicas y estrategias que nortean las orientaciones pedagógicas y metodológicas. Las personas sordas, hablantes de lengua de señas, no son la excepción a estas realidades, por lo que las reflexiones encuadradas en este campo, son de interés.

La enseñanza, aprendizaje y dominio de las lenguas escritas por parte de personas sordas ha generado atención académica desde la publicación del primer estudio linguiístico de la lengua de señas norteamericana de William Stokoe en 1965. Sin embargo, actualmente, existen ausencias en lo referente a como estos procesos son dados y deben ser atendidos en las aulas de clase. Existen varias creencias referentes a la imposibilidad de aprendizaje de una lengua escrita - de carácter oral - por sordos, lo que ha llevado, en muchas oportunidades a un sesgo académico, que por un lado, alienta el reforzamiento de la lengua materna y segunda desde la escuela, y por otro, aboga por los métodos oralistas usados desde la edad media. Estos escenarios animan a contemplar otras perspectivas, donde la 'naturalidad' en los 
procesos de adquisición-aprendizaje de las lenguas puede responder de forma asertiva a diversos fenómenos lingüísticos. La cuestión a tratar en las próximas líneas está enmarcada en identificar aspectos que influyen en el aprendizaje de la lengua inglesa escrita por parte de escolares sordos, y como estos se distancian o acercan a los procesos observados en las clases de español escrito como segunda lengua.

\section{Procesos históricos de enseñanza de lenguas para sordos}

El proceso de enseñanza de las lenguas orales para las personas con deficiencia auditiva ha sido una tarea de la que siempre se ha responsabilizado la escuela. Juan Pablo Bonet (finales del siglo XVI), inició un curioso estudio fonético de la lengua española que fue compendiado en su libro Reduction de las letras (1620), en el cual dedicó un apartado a los sordos llamado, Arte para enseñar á ablar los Mudos (OVIEDO, 2006). En esta obra, el escritor desarrolló una metodología de enseñanza y asimilación de los sonidos reducidos a la mera representación gráfica de la letra. De esta forma sentó las bases de lo que sería prioridad, por muchos años, en lo que a la educación de sordos se refiere: la lengua oral.

Ya en el en el siglo XVIII con Michael de L'Epèe, se comienza a gestar lo que se denominaría la escuela para sordos. Fundó la primera institución especializada en personas que no escuchaban, cuyo medio de comunicación eran señas rudimentarias y, algunos signos metódicos para la enseñanza del francés escrito. Publicó dos obras, Institution des sourds-muets par la voie des signes méthodiques ${ }^{3}$ (1776) y La véritable manière d'instruire les sourds et muets, confirmée par une longue expérience ${ }^{4}$ (1784). De L'Epèe dio un gran salto al considerar las convenciones diarias de comunicación entre los sordos y sus familias como señas naturales, y aquellas que precisaban ser creadas para un saber técnico señas metódicas, generando así la idea de que es posible concebir vínculos con el mundo a través de la lengua. En este sentido, aquella que puede ser articulada de forma natural, sin tener que pasar por procesos dolorosos y antinaturales en su aprendizaje.

Adelantándonos un poco en el tiempo, y llegando al siglo XX, la cuestión de utilizar lenguas de señas en las Instituciones era algo que aún resonaba en las discusiones académicas. Las lenguas de señas continuaban siendo consideradas como sistemas de comunicación empobrecidos, que solo posibilitaban interacciones básicas y de carente contenido semántico

${ }^{3}$ La instrucción de los sordomudos a través de las señas metódicas.

${ }^{4}$ La verdadera manera de educar a los sordomudos, confirmada por una larga experiencia. 
y pragmático. Fue el mismo descubrimiento de lo contrario, en la primera mitad del siglo XX, lo que llevó al profesor emérito de la prestigiosa Universidad de Gallaudet, William Stokoe, a publicar su monografía Sign language structure ${ }^{5}$ (1960) y, poco después, el primer diccionario de la lengua de señas estadounidense elaborado con criterios lingüísticos en 1965.

En la década de los 80 s y los 90s, las comunidades de sordos en Colombia comenzaron a participar de estudios lingüísticos de su propia lengua, lo que la hizo visible, aparte de presentarlos como sujetos de derechos bajo una visión socioantropológica. Personas que comparten una lengua y una cultura en común. De estos esfuerzos surge, años más tarde, “Apuntes para una gramática de la lengua de señas colombiana" (2001) de Alejandro Oviedo, un tratado lingüístico que buscó describir la lengua de señas, así como posibilitar estudios posteriores.

Llegando a nuestros días, y con una visión más clara de lo que es la lengua de señas para el sordo, se plantea un reto que ha estado en boga desde la incursión de los sordos en la educación secundaria y universitaria a comienzos del siglo XXI: la enseñanza del español como lengua escrita. Este reto, aún presente en las Instituciones Educativas, no cuenta con pesquisas que orienten su enseñanza, lo que deja tras de sí bastantes preguntas en lo relacionado con los procesos envueltos en su enseñanza y aprendizaje. En este artículo no vamos a responder esta cuestión, pero sí vamos a abrir la reflexión a partir de los datos recolectados en las aulas de inglés escrito para sordos, aulas pensadas y adaptadas desde la perspectiva de prácticas translíngues.

\section{La perspectiva de prácticas translingües}

Tradicionalmente se ha conocido al término bilingüismo como la habilidad o manejo de dos o más lenguas. Muchos teóricos han discrepado al respecto, basándose algunos, en las experiencias de uso de las lenguas en diversos contextos, y otros, en el uso de ellas en el mismo; aparte de los procesos cognitivos, lingüísticos, y sociales involucrados en este proceso.

Partiendo de estas visiones tradicionales, García y Wei (2014, p. 47-48, traducción propia) mencionan que "las nociones tradicionales de bilingüismo y multilingüismo son adictivas, o sea, los hablantes son condicionados a adicionar lenguajes autónomos completos o incluso pequeñas estructuras parciales de esos lenguajes". Las lenguas han sido 
consideradas como sistemas autónomos en el sujeto bilingüe, capaces de responder a necesidades específicas y a determinados contextos. El poco o mal uso de ellas está medido dentro de un sistema de gramatical, relegando factores ambientales y de interlocución lingüística a otro plano.

Los citados autores rescatan el término "bilingüismo dinámico" de Wallace Lambert (1974). Este, contrario a la concepción de lenguas que se adhieren de forma autónoma al cerebro del aprendiz y utilizadas en contextos específicos, el bilingüismo dinámico "sugiere que las prácticas de lenguaje de los bilingües son complejas e interrelacionadas; ellas no emergen de forma lineal o funcionan separadamente" (2014, p. 50, traducción propia). Es decir, esta propuesta dinámica resalta la interrelación de las lenguas habladas por el bilingüe con fines lingüístico-comunicativos.

Suresh Canagarajah (2013) presenta el concepto de prácticas translingües como un integrador de recursos en repertorios linguiísticos. Rompe con la idea tradicional de delimitación lingüística y amplía el significado vinculando aspectos presentes en el proceso comunicativo. En este caso, los bilingües, como podemos considerar a nuestros estudiantes sordos, producen la lengua escrita partiendo de un banco léxico compuesto por varias lenguas, dando origen a movimientos transformativos, "generando nuevos significados y gramáticas (Ibídem, p. 16, traducción propia).

\section{Metodología}

La investigación presentada discute el proceso de aprendizaje del inglés escrito como lengua extranjera a partir de una visión de lengua oral para estudiantes con una lengua visogestual-espacial como primera. Las relaciones lingüísticas que se dan entre ambas lenguas, de asimilación y aceptación, van a estar mediadas por el español, lengua obligatoria dentro del currículo académico como segunda. Por lo tanto, los resultados obtenidos van a revelar qué aspectos facilitan el aprendizaje del inglés en comparación con la lengua española. A su vez, se hará una discusión sobre la construcción de sentidos basada en la propuesta de prácticas translíngues de Suresh Canagarajah (2013).

Las intervenciones que sirvieron de escenario en el análisis fueron realizadas dentro de la institución educativa con la licencia y supervisión de la coordinación general. Se llevaron a cabo 20 sesiones semanales durante un semestre académico. La metodología usada incluyó clases bajo un abordaje de escritura y visual participativo. Los materiales de análisis fueron 
dados gracias a la consignación de eventos observados en el aula y registrados en grabaciones y apuntes, como por los diferentes ejercicios y evaluaciones aplicados a los estudiantes. Asimismo, se hizo un proceso de observación y recolección de producciones hechas en esta lengua, como también entrevistas y encuestas.

\section{Resultados}

A partir de los datos arrojados por las actividades, grabaciones y encuestas hechas en las intervenciones, rescatamos material elemental para la caracterización de las prácticas de aprendizaje dadas en los sujetos de investigación. A continuación se presentan las diferentes delimitaciones dadas en el análisis para luego ser analizadas con sus respectivas conclusiones. El trabajo fue organizado en categorías siguiendo el siguiente orden de trabajo: construcciones básicas en español e inglés, información personal, pronombres interrogativos y descripciones.

\section{Construcción de oraciones en español}

Para la gran mayoría de los estudiantes sordos intervenidos, las clases de español representan más una obligación institucional que una oportunidad para su aprendizaje y aprovechamiento. Desde el inicio de este trabajo, nos manifestaron que su desempeño en esta lengua no era el mejor y que querían intentar con el inglés. Por esta razón, consideramos conveniente recolectar este sentir a través de la pregunta: ¿Cómo consideran su producción escrita en español?, y ¿Por qué? Este ejercicio nos permitió por un lado, consignar sus razones $y$, por otro, analizar la forma en que organizan las ideas en español.

A continuación se presentan dos de las seis muestras:

1. ¿Cómo consideran su producción escrita del español?, y ¿por qué?

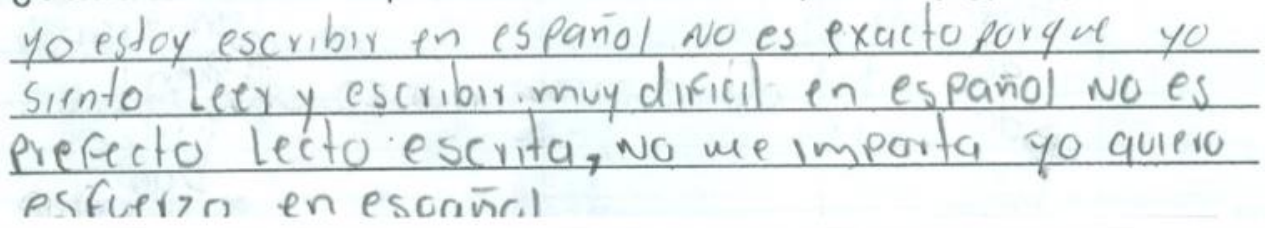


1. ¿Cómo consideran su producción escrita del español?, y ¿por qué? No perfecta escrita del español pero siempre
algun menor el articulo y frase muy diferente
porgue soy idioma pasado muy tarde lecto escrita.
no enseñando siempre Primero lengua de señas.

Las dos muestras revelan que ellos consideran al español una lengua de gran dificultad, con diferencias marcadas en comparación con su lengua, y cuya aproximación y aprendizaje a ella se hizo de forma tardía. A este respecto, Baquero (2003, p. 31) comenta que un oyente "ha adquirido [el español] mediante el canal auditivo en la interacción con la familia y con el medio social"; mientras que el sordo lo hace dentro de los procesos escolares y en otros tiempos. En este sentido, la asimilación de la lengua escrita dentro de su proceso educativo responde a otras lógicas y se desarrolla en otras etapas, lo que la hace parecer "difícil”.

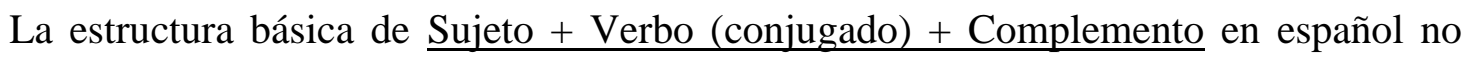
está presente en la organización escrita de las muestras, sino que, responden a lo concluido por Chilito (2010, p. 29): "deaf students present a specific way to organize the sentence in Colombian Sign Language. They use the structure of Subject + Object + Verb which is different in Spanish" otro esquema, supone un reto para su producción en español, restándoles el interés y, más bien, terminar organizándolas en la estructura de su propia lengua sin seguir los componentes sintácticos y morfológicos.

\section{Construcción de oraciones básicas en inglés}

Siguiendo los parámetros del nivel A1 del Marco Común Europeo de Referencia para las Lenguas ${ }^{7}$ se introdujeron los tópicos "personal introduction" "verb to be" y "personal and interrogative pronouns". La asimilación se hizo de forma relativamente rápida. Se realizaron

${ }^{6}$ Los estudiantes sordos presentan una forma específica de organizar oraciones en lengua de señas colombiana. Ellos usan la estructura de Sujeto + Objeto + Verbo la cual es diferente en español.

7 (CONSEJO DE EUROPA, 2002, p. 30):

Niveles comunes de referencia: cuadro de autoevaluación.

Escribir. Expresión escrita:

- Soy capaz de escribir postales cortas y sencillas; por ejemplo, para enviar felicitaciones. Sé rellenar formularios con datos personales; por ejemplo, mi nombre, mi nacionalidad y mi dirección en el formulario del registro de un hotel. 
varias actividades con respectivas retroalimentaciones en las que ellos mismos se corregían. A continuación se presentan diferentes muestras de este trabajo:

Información personal

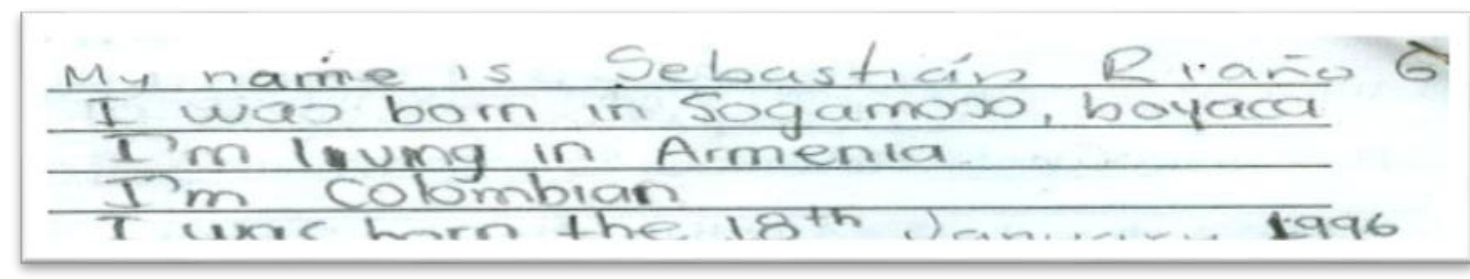

beccuse I Inke tolstudy Lnglish.
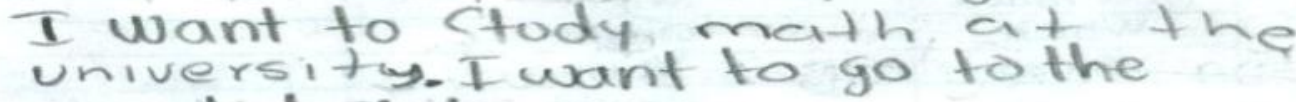

united Stater

En estas, se observa buen uso del verbo "to be" tanto en presente como en pasado. Además el orden estructural de las oraciones sigue la estructura de la lengua, algo que no aconteció en su producción en español (la cual presentaba alteraciones de orden sintáctico y de concordancia), a pesar de que el inglés maneja su misma estructura. A diferencia de la estructura de la lengua de señas, observada por Chilito (2010) de Subject + Object + Verb, en este caso no interfirió en su escritura en inglés.

Pronombres interrogativos

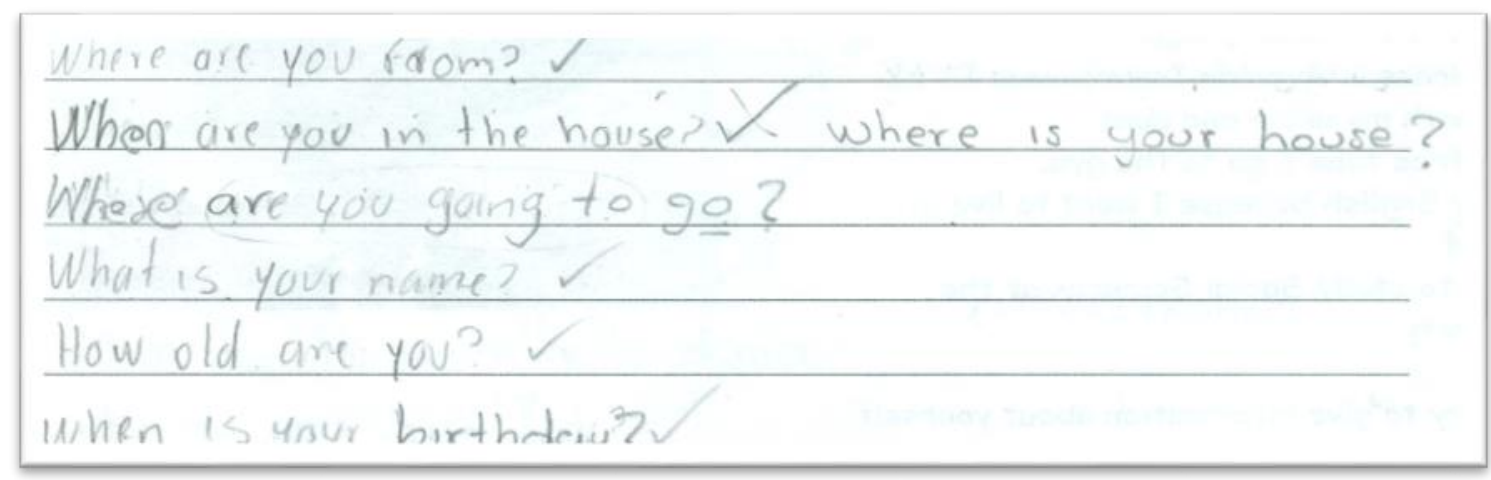

Esta práctica exigió la enseñanza del uso del verbo "to be" como auxiliar. Hubo muchas preguntas respecto a qué sucedería si el orden se alteraba, ya que, como ellos puntuaban, el español les permite ubicarlos en diferentes partes de la oración. La explicación del por qué la alteración de esta estructura afectaría la connotación semántica de las oraciones hizo que los estudiantes escribieran pensando en una regla específica. Hallaron lógico seguir una estructura que respete un orden sistemático. Esta muestra permite ver que existe un 
seguimiento de la regla Pronombre interrogativo + Verbo auxiliar + Pronombre personal + Complemento + Signo de pregunta.

Descripciones

Estos ejercicios fueron realizados en la pizarra con la ayuda de apoyos visuales que permitieron observar las imágenes que iban a ser descritas. El objetivo principal era contrastar la colocación de las oraciones en ambas lenguas, generando reflexiones metalingüísticas que respondieran a una lógica organizacional. Primero se hicieron descripciones en español y luego en inglés. Con base en los siguientes trabajos se detallan las imágenes presentadas: En español

\section{Yo ver hombre rojo camisa caminar después encontrar mujer bonito}

\section{Cuando negro mujer encontrar hombre rojo ropa ella asustado}

Se observa uso de los verbos en infinitivo en lugar de verbos conjugados. Por ejemplo: "yo ver" en lugar de yo veo; y "mujer encontrar" en lugar de mujer encuentra. Los verbos en infinitivo guardan un significado propio que no requiere variación en las diferentes personas, es decir, no requiere modificaciones para ser entendido. Baquero (2003), refiriéndose a los estudios hechos por Hatober y Robbins (1980 y 1981), menciona que se han encontrado dificultades en la "comprensión sintáctica de los sordos" (32) en lo que a la lectura se refiere. Este aspecto guarda relación con las muestras expuestas, su lógica organizacional responde a otros patrones, por lo que se basan en ellos para producir significados en otra lengua, en este caso, el español escrito.

Por otro lado, el uso de los géneros en los adjetivos utilizados no guarda concordancia. "Rojo camisa"; "mujer bonito"; "negro mujer", así lo demuestran. Chilito (2010, p. 35) lo detectó en su estudio, y menciona que "Colombian Sign Language is a language which is neutral in relation to gender. [...] Of course this neutralism of gender influences the written production in Spanish"8. Los estudiantes siguen una secuencia lógica basada en su lengua que no discrimina géneros ni categoriza los verbos en personas.

${ }^{8}$ La lengua de señas colombiana es una lengua que es neutral en relación con el género. [...] Por supuesto este neutralismo de género influencia la producción escrita en español. 
Ahora veamos la contraparte. Cuando les pedimos que trataran de describir la misma imagen de la mujer que se encontraba con el hombre de rojo en inglés, previo estudio de vocabulario y estructura, lo hicieron de esta forma:

En inglés

\section{The man red t-shirt walks to find the beautiful woman}

When blackwoman finds the man red clothes she afraid

Estas muestras revelan una secuencia estructural de Sujeto + Verbo + Complemento, demostrando ser un aspecto que no representa dificultad de uso. Por su parte, los adjetivos "beautiful" y "black" son ubicados antes del sustantivo, siguiendo un orden lógico previamente explicado. En el caso del español se observó una bimodalidad, al ubicarlo antes y después del sustantivo. Parece ser que se presenta una dificultad al combinar aspectos morfosintácticos en una sola oración, afectando alguna parte de la misma. En este caso, de acuerdo con lo presentado por Chilito (2010), la lengua de señas se aproxima al inglés en lo referente al uso de los géneros y la forma. Baquero (2003) en el análisis de los recursos lingüísticos de español escrito de uno de los escolares sordos intervenidos en su investigación, diferente a lo observado en la producción en inglés anterior, encontró que "Las oraciones [no] siguen las reglas gramaticales (morfosintácticas) del castellano [...] existen intentos oracionales en oraciones simples [...] en el texto [...] adjetivos calificativos, adverbio de cantidad que pueden aparecer a veces sin su valor sino como meras formas." (57)

Por último, la conjugación de las terceras personas del singular en inglés no representó dificultad. Desde el comienzo manifestaron adaptabilidad a esta característica del inglés, argumentando que existe menos dificultad en comparación con el español.

\section{Considerações finais}

El análisis de los resultados obtenidos en los seis estudiantes intervenidos en esta pesquisa señala una diferencia en la producción escrita entre el inglés y el español. A pesar de que los estudiantes han estudiado por más de 10 años lengua escrita (español) como parte del currículo académico, su producción en esta lengua revela un fuerte distanciamiento de sus 
estructuras y reglas. En contraste, la producción en inglés escrito nivel A $1^{9}$ revela un manejo de sintaxis y forma más correcto. Sin embargo, es de resaltar que estos resultados son contrarios a los de Taylor (1969, apud Baquero, 2003, p. 33), quien encontró que la producción escrita de sordos adolescentes, no seguía "las reglas gramaticales del inglés; tenían problemas con los determinantes, los verbos auxiliares, con las frases de relativo y con oraciones complejas." En este sentido, podríamos pensar no solo en términos de gramáticas similares que facilitan el aprendizaje del inglés (ya que si ese fuera el caso, los estudiantes intervenidos por Taylor, no presentarían ninguna dificultad), sino en otros aspectos que facilitan su asimilación y manejo.

Lo que los estudiantes piensan con relación a su producción escrita en las dos lenguas marca una diferencia. Ellos culpan a las complejidades morfosintácticas del español y a su estudio y uso tardío como determinantes en su producción. Su contexto familiar preescolar no exigía el uso de la lengua escrita, y su comunicación se basaba en convenciones señadas caseras. Es decir, se utilizaba un léxico demostrativo que respondía a necesidades propias del momento. Su acercamiento a una estructura se hizo al aprender la lengua de señas colombiana, en donde tuvieron que seguir unas ordenaciones que respetaban una sintaxis y una forma. Esta primera referencia gramatical crea una fuerte conexión con la producción en otras lenguas. (Chilito, 2010); (Baquero; 2003). El sentir de "errar" siempre que escribían español y las contantes correcciones en la escuela, creó un desacato a las normas del español, prefiriendo usar la estructura de su lengua. Este punto motivacional podría responder la antítesis mostrada en el párrafo anterior, en el que se demostró que el acercamiento gramatical no es el componente decisivo del manejo del inglés en nuestro caso.

Aunque no podemos negar la tranquilidad expresada por los estudiantes al darse cuenta de algunas homogeneidades entre su lengua y el inglés, este hallazgo nos lleva a concluir en el respeto de un orden jerárquico dentro del proceso de aprendizaje, consignado en las siguientes premisas:

1. La motivación de los estudiantes como dinamizador de asimilación de otras estructuras lingüísticas.

2. La creación de un repertorio multilingüe que responde a un único repertorio comunicativo escrito.

3. La asimilación de algunas estructuras en su lengua materna con el inglés escrito y no con el español.

${ }^{9}$ (Consejo de Europa, 2002) Marco común europeo de referencia para las lenguas. 
Esta jerarquía propuesta obedece a una reflexión pedagógica, ocupando el primer lugar como acondicionadora de ambientes de aprendizaje que facilitan la asimilación de nuevos conocimientos. Por otro lado, el banco multilingüístico creado es capaz de responder a diversas estructuras de las lenguas, lo que permite buscar conexiones entre lo que han aprendido y lo que están aprendiendo. En este caso, el inglés es asimilado de forma asertiva al estar mediada su enseñanza por una práctica pedagógica atrayente, y diferente a la experiencia con el español, de acuerdo a sus experiencias y comentarios.

A pesar de que los estudiantes no son conscientes de esos procesos, sus prácticas muestran que las lenguas y gramáticas aprendidas son parte de su uso escrito diario, generando nuevos sentidos en su mundo bilingüe.

\section{REFERENCIAS}

BAQUERO, S. ¿Cómo narran los escolares limitados auditivos colombianos? Portal de revistas UN, Bogotá, n. 16, p. 30-97, 2003.

BENVENISTE, É. Problemas de Lingüística Geral. Tradução de Maria da Glória Novak e Luiza Neri. San Pablo: Ed. Nacional; Ed. da Universidade de São Paulo, 1976.

CHILITO, J. The Influence of CSL on Spanish (proyecto de grado). Armenia: Universidad del Quindío, 2010.

CONSEJO DE EUROPA. Marco común europeo de referencia para las lenguas. Madrid: Ministerio de Educación, Cultura y Deporte, Subdirección General de Cooperación Internacional, para la edición impresa en español, 2002.

GARCÍA, O; WEI, L. Translanguaging: Language, Bilingualism and Education. Hampshire: Palgrave Macmillan, 2014.

OVIEDO, A. Apuntes para una gramática de la lengua de señas colombiana. Cali: Universidad del Valle / INSOR, 2001.

OVIEDO, A. La vida y la obra de Juan Pablo Bonet (1573-1633). Cultura Sorda, 2006. Disponible en: <http://www.cultura-sorda.org/juan-pablo-bonet/> Acceso el: 16 julio 2017. 


\section{Como referenciar este artículo}

BECERRA-CALLERO, Jorge Armando.; ROJAS-ISAZA, Angélica María. Enseñanza del inglés para sordos desde una perspectiva de prácticas translingües: motivaciones e intereses. Rev. EntreLínguas, Araraquara, v. 4, n. 2, p. 205-217, jul./dez., 2018. E-ISSN: 2447-3529. DOI: 10.29051/rel.unesp.v4.n2.2018.11619

Submetido em: 31/07/2018

Aprovado em: 16/10/2018 